\title{
Fentanyl Delays the Platelet Inhibition Effects of Oral Ticagrelor: Full Report of the PACIFY Randomized Clinical Trial
}

\author{
Khalil Ibrahim ${ }^{1, *}$ Rohan Shah ${ }^{2, *}$ Rakesh R. Goli ${ }^{2}$ Thomas S. Kickler ${ }^{3}$ William A. Clarke ${ }^{3}$ Rani K. Hasan ${ }^{1}$ \\ Roger S. Blumenthal ${ }^{1,4}$ David R. Thiemann ${ }^{1}$ Jon R. Resar ${ }^{1}$ Steven P. Schulman ${ }^{1}$ John W. McEvoy ${ }^{1,4}$
}

${ }^{1}$ Division of Cardiology, Johns Hopkins University School of Medicine, Baltimore, Maryland, United States

2 Department of Medicine, Johns Hopkins University School of Medicine, Baltimore, Maryland, United States

${ }^{3}$ Department of Pathology, Johns Hopkins University School of Medicine, Baltimore, Maryland, United States

${ }^{4}$ Ciccarone Center for the Prevention of Heart Disease, Johns Hopkins University School of Medicine, Baltimore, Maryland, United States

\begin{abstract}
Address for correspondence John W. McEvoy, MB, BCh, BAO, MEHP, MHS, Division of Cardiology and Johns Hopkins Ciccarone Center for the Prevention of Heart Disease, Johns Hopkins University School of Medicine, 600 N. Wolfe Street, Blalock 524C, Baltimore, MD 21287, United States (e-mail: jmcevoy1@jhmi.edu).
\end{abstract}

Thromb Haemost 2018;118:1409-1418.

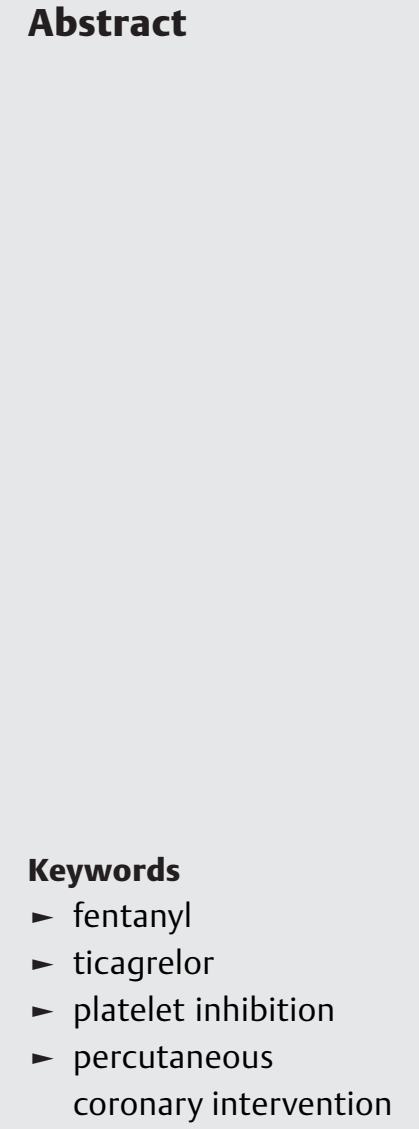

Morphine delays oral $\mathrm{P}_{2} \mathrm{Y}_{12}$ platelet inhibitor absorption and is associated with adverse outcomes after myocardial infarction. Consequently, many physicians and first responders are now considering fentanyl as an alternative. We conducted a single-centre trial randomizing cardiac patients undergoing coronary angiography to intravenous fentanyl or not. All participants received local anaesthetic and intravenous midazolam. Those requiring percutaneous coronary intervention $(\mathrm{PCl})$ with stenting received $180 \mathrm{mg}$ oral ticagrelor intraprocedurally. The primary outcome was area under the ticagrelor plasma concentration-time curve ( $A \cup C_{0-24}$ hours). The secondary outcomes were platelet function assessed at 2 hours after loading, measured by $\mathrm{P}_{2} \mathrm{Y}_{12}$ reaction units (PRUs) and light transmission platelet aggregometry. Troponin-I was measured post-PCI using a high-sensitivity troponin-I assay (hs-Tnl). All participants completed a survey of pain and anxiety. Of the 212 randomized, 70 patients required coronary stenting and were loaded with ticagrelor. Two participants in the no-fentanyl arm crossed over to receive fentanyl for pain. In as-treated analyses, ticagrelor concentrations were higher in the no-fentanyl arm ( $A \cup C_{0-24}$ hours $70 \%$ larger, $p=0.03$ ). Platelets were more inhibited by 2 hours in the no-fentanyl arm ( 71 vs. 113 by PRU, $p=0.03$, and $25 \%$ vs. $41 \%$ for adenosine diphosphate response by platelet aggregation, $p<0.01$ ). Mean hs-Tnl was higher with fentanyl at 2 hours post-PCI $(11.9 \mathrm{vs.} 7.0 \mathrm{ng} / \mathrm{L}, p=0.04)$ with a rate of enzymatic myocardial infarction of $11 \%$ for fentanyl and $0 \%$ for no-fentanyl $(p=0.08)$. No statistical differences in self-reported pain or anxiety were found. In conclusion, fentanyl administration can impair ticagrelor absorption and delay platelet inhibition, resulting in mild excess of myocardial damage. This newly described drug interaction should be recognized by physicians and suggests that the interaction between opioids and oral $\mathrm{P}_{2} \mathrm{Y}_{12}$ platelet inhibitors is a drug class effect associated with all opioids.

Clinical Trial Registration: https://clinicaltrials.gov/ct2/show/NCT02683707 (NCT02683707).

* Khalil Ibrahim and Rohan Shah contributed equally to the article.

received

March 21, 2018

accepted after revision June 3, 2018
DOI https://doi.org/

10.1055/s-0038-1666862. ISSN 0340-6245. (c) 2018 Georg Thieme Verlag KG Stuttgart · New York
License terms

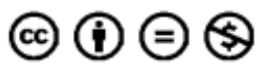




\section{Introduction}

Fentanyl is a powerful and rapid-acting intravenous opioid used in up to $90 \%$ of percutaneous coronary intervention (PCI) cases in North America. ${ }^{1}$ Fentanyl is commonly administered with subcutaneous lidocaine and intravenous midazolam to achieve sedation and analgesia at the start of the $\mathrm{PCI}$ procedure and as needed throughout the case. Given concerns that morphine is associated with adverse outcomes among myocardial infarction patients, ${ }^{2}$ many emergency room providers are also now considering fentanyl for the treatment of acute chest pain in these patients. However, practices differ outside the United States with, for example, some studies reporting that less than $40 \%$ of non-U.S. cardiologists use any sedation during PCI. ${ }^{1}$ Indeed, many European centres rarely if ever provide opioids for $\mathrm{PCl}^{3}$ It is therefore surprising that, despite the wide geographic variation in opioid use for $\mathrm{PCI}$, no prospective randomized trials have been performed to evaluate the efficacy and safety of intravenous fentanyl in the setting of PCI.

The assumption that fentanyl is safe for $\mathrm{PCI}$ is challenged by recent research demonstrating an interaction between intravenous morphine and oral $\mathrm{P}_{2} \mathrm{Y}_{12}$ platelet inhibitors. ${ }^{2}$ Morphine significantly delays the gastrointestinal absorption of oral $\mathrm{P}_{2} \mathrm{Y}_{12}$ inhibitors, which results in delayed platelet inhibition in healthy controls and acute coronary syndrome (ACS) patients. ${ }^{4-10}$ The presumed mechanism is slowed gastrointestinal motility due to opioid-induced activation of mu receptors. ${ }^{2,11}$ Given the critical need for rapid platelet inhibition among stable coronary artery disease (CAD) and ACS patients undergoing $\mathrm{PCI}$, it is important to understand whether fentanyl has a similar effect to morphine on $\mathrm{P}_{2} \mathrm{Y}_{12}$ inhibitor absorption. The importance of filling this gap in knowledge is heightened by the following two considerations: (1) the pharmacokinetics of these two opioids differ substantially and one cannot assume the more rapid-acting fentanyl will also delay $\mathrm{P}_{2} \mathrm{Y}_{12}$ inhibitor absorption; and (2) the clinical implications differ as fentanyl is widely administered in U.S. catheterization laboratories (whereas morphine is given less frequently, for example, fewer than $30 \%$ of U.S. non-ST elevation myocardial infarction patients receive morphine during their hospital admission ${ }^{12}$ ).

Our group recently reported top-line results from the Platelet Aggregation with Ticagrelor Inhibition and Fentanyl (PACIFY) randomized trial, which examined whether routine use of fentanyl in the cardiac catheterization laboratory delays the absorption and action of a $180-\mathrm{mg}$ oral load of ticagrelor given to patients with CAD undergoing clinically indicated ad hoc PCI. ${ }^{13,14}$ This recent report provided limited results for the pre-specified primary and secondary intention-to-treat endpoints, demonstrating significantly lower blood concentrations of ticagrelor over the 24 hours after loading among those randomized to fentanyl and, consequently, less platelet inhibition at 2 hours after loading. ${ }^{14}$ However, the full complement of results from the PACIFY trial have not been published to date. Herein, we now report baseline demographic and procedural characteristics of the study sample, important results from as-treated analyses (there was crossover from the no-fentanyl to fentanyl arms) and extended results for the platelet function, high-sensitivity troponin-I (hs-TnI) and self-reported patient comfort outcomes.

\section{Methods}

\section{Study Design}

The PACIFY trial was a single-centre randomized trial enrolling adults scheduled to undergo clinically indicated coronary angiography and randomizing them to receive fentanyl or no fentanyl during the procedure (see - Fig. 1 for trial Consolidated Standards of Reporting Trials flow diagram). Full details of the trial design, including inclusion and exclusion criteria, have been published previously. ${ }^{13}$ Randomization was performed in blocks of 4 with an allocation ratio of $1: 1$. The study protocol was registered in February of 2016 (NCT02683707) and no protocol changes were made thereafter. The protocol was approved by the Johns Hopkins Institutional Review Board and all participants provided written informed consent before the angiogram procedure.

\section{Study Procedures}

Participants were enrolled at the Johns Hopkins Hospital between 20th April 2016 and 25th May 2017. All participants received subcutaneous lidocaine and intravenous midazolam at the start of the procedure and as needed thereafter. Doses were at the discretion of treating providers. Patients who were randomized to intravenous fentanyl received this (in addition to lidocaine and midazolam) at the start of the procedure and as needed thereafter, with doses also determined by the treating provider.

Patients were not informed of their randomized allocation (i.e. no-fentanyl or fentanyl) by the study team. Outcomes assessors were also blinded. However, because medications were administered by nursing staff at the request of the interventional cardiologist, treating providers were not blinded. Of note, participating cardiologists could provide fentanyl during the case, irrespective of randomized allocation, if deemed clinically necessary for bail-out treatment of uncontrolled pain. All participating patients completed a selfreported survey of pain and anxiety 2 hours after the end of the procedure.

Because the research team was almost always unaware of coronary anatomy at the time of patient enrollment prior to angiography, only a subset of those enrolled and randomized (70 of 212 participants) underwent ad hoc PCI for clinically indicated treatment of obstructive CAD. The others were either treated medically or referred for surgery. Participants who had PCI were given an oral loading dose of $180 \mathrm{mg}$ of ticagrelor at the conclusion of diagnostic angiography, immediately prior to PCI. Per usual practice in our catheterization laboratory, tablets were not crushed (this is relevant because prior studies have shown that crushed ticagrelor results in earlier platelet inhibition). ${ }^{15}$ While its use in elective $\mathrm{PCI}$ is off label, ticagrelor was the chosen $\mathrm{P}_{2} \mathrm{Y}_{12}$ 


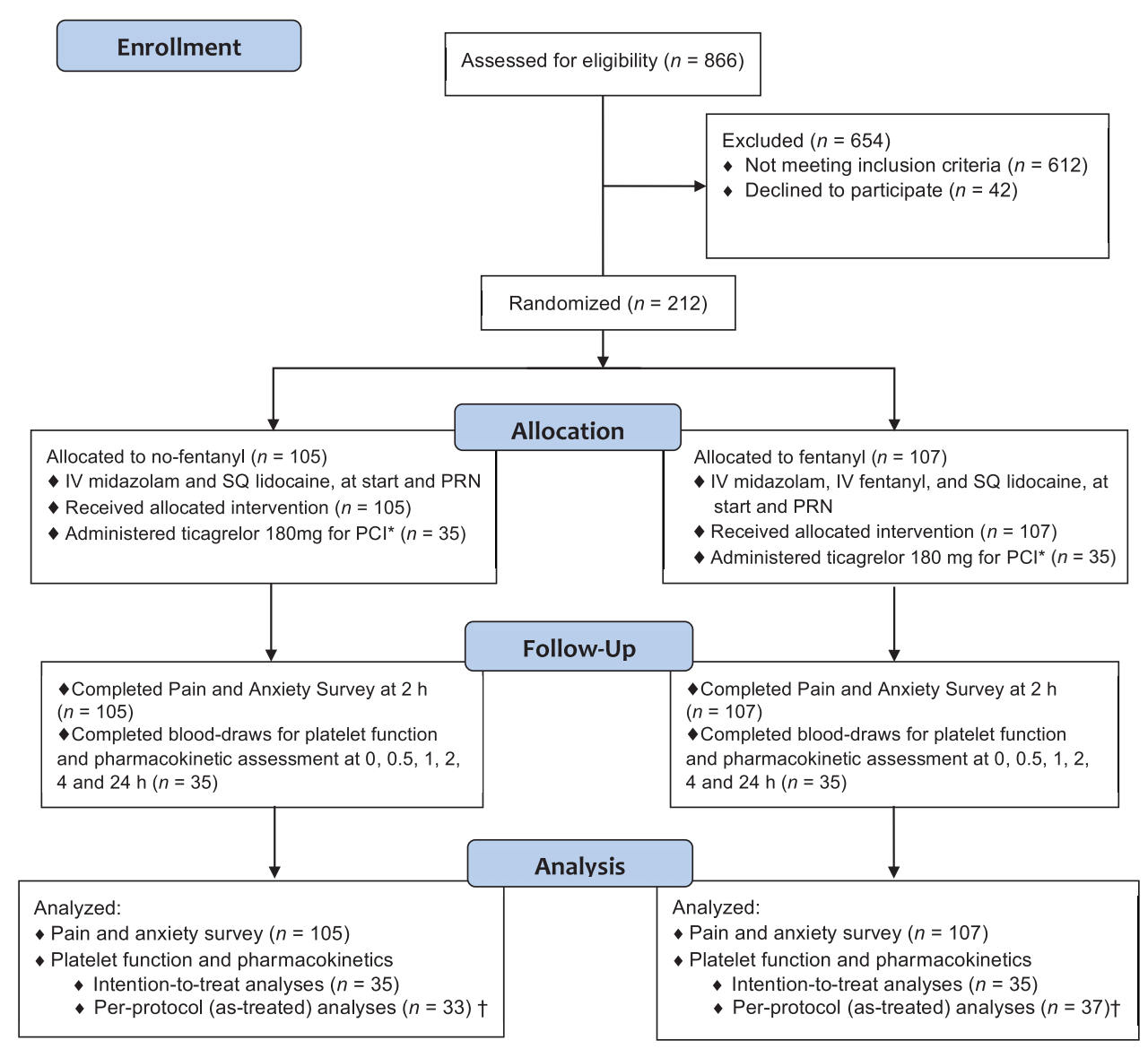

Fig. 1 Platelet Aggregation with Ticagrelor Inhibition and Fentanyl (PACIFY) Consolidated Standards of Reporting Trials (CONSORT) diagram. ${ }^{*}$ Ticagrelor was administered orally during the catheterization procedure at the time percutaneous coronary intervention (PCI) was determined to be necessary. †Two participants in the randomly allocated to the no-fentanyl arm received intravenous (IV) fentanyl for pain within 30 minutes of ticagrelor loading and were analysed as having received fentanyl in as-treated analyses. PRN, pro re nata (as needed); SQ, subcutaneous.

agent for this study because it achieves more rapid and complete platelet inhibition, theoretically negating the need for pre-treatment hours prior to the procedure and also because this is the $\mathrm{P}_{2} \mathrm{Y}_{12}$ agent of choice for ACS. ${ }^{16}$ Participants were required to be fasting prior to the procedure. Among patients who had PCI, baseline blood samples were collected prior to ticagrelor loading and at the following time intervals thereafter: $0.5,1,2,4$ and 24 hours postloading. The decision about chronic post-PCI dual antiplatelet therapy was at the discretion of the treatment team; patients continuing ticagrelor received a maintenance dose at 12 hours post-loading and patients being switched to an alternative were reloaded with the new $\mathrm{P}_{2} \mathrm{Y}_{12}$ agent a minimum of 12 hours and maximum of 24 hours after the initial ticagrelor load. ${ }^{17}$

\section{Pharmacokinetic Assessment}

Ticagrelor plasma concentrations were measured by blinded assessors using liquid chromatography-tandem mass spectrometry at $0.5,1,2,4$ and 24 hours after the load. Because persons with $\mathrm{P}_{2} \mathrm{Y}_{12}$ inhibitor use within 14 days were excluded, we presumed baseline (pre-loading) ticagrelor concentration was zero. Samples were processed using protein precipitation with methanol containing deuterating ticagrelor internal standard (ALSACHIM, France). After centrifugation, the supernatant was transferred to autosampler vials and then quantified. Calibration curves were prepared using blank plasma samples spiked with ticagrelor standards (ALSACHIM). Further details are presented in the -Supplementary Material (available in the online version). Analysis was performed using a Thermo Fisher Vanquish UHPLC system coupled with a Thermo Fisher TSQ Vantage QqQ mass spectrometer.

\section{Pharmacodynamic (Platelet Function) Assessment}

Platelet function was measured by blinded assessors at baseline (pre-loading), 0.5, 1, 2, 4 and 24 hours after ticagrelor administration. Platelet function at each of these time points was measured as $\mathrm{P}_{2} \mathrm{Y}_{12}$ Reactivity Units (PRUs) with the VerifyNow system (Accriva Diagnostics, San Diego, California, United States). We also assessed platelet function at 2 hours using light transmission platelet aggregometry. Platelet aggregometry was performed with adenosine diphosphate (ADP) agonism using a Chrono-Log Model 560CA aggregometer (Chrono-Log, Havertown, Pennsylvania, United States). The maximum aggregation response within 
5 minutes was recorded as percentage increase from baseline. All platelet function tests were conducted in duplicate and the intra-individual coefficient of variation was $2 \%$ for PRU and 3\% for light aggregometry. For the below reporting of platelet function outcomes, we excluded the single participant in the fentanyl arm who received a glycoprotein IIb/ IIIa antagonist during their procedure.

\section{High-Sensitivity Troponin Measurement}

Myocardial injury was assessed using a research-only hs-TnI assay at 2 hours post-PCI, with a limit of detection of $1.2 \mathrm{ng} / \mathrm{L}$ and a 99th percentile (upper reference limit) of $26.2 \mathrm{ng} / \mathrm{L}$. TnI levels were also available in a non-random sub-group at 4 hours post-PCI.

\section{Study Outcomes}

The pre-specified primary outcome was ticagrelor blood concentration during the 24 hours after loading, as measured by the area under the plasma concentration-time curve $\left(A_{U} C_{[0-24]}\right)$. Pre-specified secondary endpoints included: (1) platelet inhibition assessed by VerifyNow (PRU) at 2 hours; (2) platelet inhibition assessed by platelet aggregometry at 2 hours; and (3) self-reported maximum pain experienced during the procedure on a 0 to 10 numeric scale.

We also compared the fentanyl and no-fentanyl groups for proportion of patients with high platelet reactivity (HPR) 2 hours after ticagrelor loading, defined both as PRU $\geq 235$ by VerifyNow $^{18,19}$ and as ADP response $\geq 46 \%$ by impedance aggregometry. ${ }^{7}$ Sensitivity analyses evaluated a HPR cut point of PRU $\geq 208$. Comparisons of PRU values at $0,0.5,1$, 4 and 24 hours were conducted as well.

In addition, we compared anxiety during the procedure and self-reported pain 2 hours after the procedure, recorded using the study survey ( - Supplementary Fig. S1, available in the online version). In a post hoc analysis, we compared maximum intra-procedural pain documented by the procedure-room nurse in the electronic health record. This assessment was done by nursing using a standard 0 to 10 -point pain scale, with 10 being the maximum pain the patient has felt.

\section{Statistical Analysis}

The sample size for this study was estimated from prior studies on morphine ${ }^{5,7}$ which suggested that 70 participants undergoing PCI would be sufficient to demonstrate significant difference in ticagrelor pharmacokinetics (at 24 hours) and pharmacodynamics (at 2 hours). Assuming that $50 \mathrm{mcg}$ of fentanyl has the same effect as $5 \mathrm{mg}$ of morphine on ticagrelor, we estimated that for an $\alpha$ of 0.05 and $80 \%$ power, 62 and 68 participants would be required to demonstrate a 40\% difference in AUC and 20\% difference in PRU at 2 hours, respectively.

Baseline characteristics were compared between randomized groups using the Fisher's exact test for proportions and, depending on the normality of data, $t$-testing or Wilcoxon rank-sum for continuous variables. When calculating the AUC, the 24-hour concentration value for patients who were continued on ticagrelor by their clinical team (and thus received a maintenance dose 12 hours after the load, $N=34$ of 70) was excluded; for analytic purposes, these 34 patients only contributed concentration values to the AUC calculation at $0,0.5,1,2$ and 4 hours. As-treated analysis compared participants according to the medication received. For these analyses, participants ( $n=2)$ who were randomized to 'nofentanyl' but who received fentanyl 30 minute before or after the ticagrelor loading dose were analysed in the fentanyl group. ${ }^{20,21}$ The level of significance was a $p$-value of $<0.05$ (two-sided). All analyses were performed using STATA 13 (Stata, College Station, Texas, United States).

\section{Results}

Of the 212 participants randomized overall, 70 required clinically indicated PCI and were loaded with $180 \mathrm{mg}$ of oral ticagrelor during the procedure ( $\mathbf{- F i g}$. 1). While all 212 participants completed the pain and anxiety survey, only the sub-group who were loaded with ticagrelor completed the full study protocol and had data for pharmacokinetic and pharmacodynamic analyses. Among the latter, baseline clinical demographics were balanced in the fentanyl and no-fentanyl groups, with no statistically significant differences in characteristics (-Table 1). Mean age was 63 years, 27\% were female and the majority were white. None of the patients enrolled presented with ACS. There were also no differences in mean cumulative midazolam exposure between groups. Fentanyl dose administration differences are reported in -Table 1. Findings were similar when we compared all 212 participants (-Supplementary Table S1, available in the online version).

\section{Extended Pharmacokinetic Results}

The intention-to-treat results of ticagrelor blood concentrations in the fentanyl and no-fentanyl groups have been previously reported ${ }^{14,22}$ and showed a marginally lower AUC $_{[0-24]}$ for ticagrelor concentration among those who received fentanyl $(p=0.05)$. These intention-to-treat data included results from two participants who crossed over from the no-fentanyl arm to the fentanyl arm within $30 \mathrm{~min}-$ utes of the ticagrelor load. In pre-specified as-treated analyses, the differences in ticagrelor concentration were more pronounced. Participants who received fentanyl had a lower $\mathrm{AUC}_{[0-24]}$ for ticagrelor concentration than those who did not (2,016 vs. 3,441 ng.h/mL, $p=0.03$ ). At each of the $0.5-, 1$ - and 2-hour time points, ticagrelor concentrations were also significantly lower among those who received fentanyl (-Fig. 2). Ticagrelor concentrations were similar in both groups at 4 and 24 hours. Although there was a trend to lower values in the fentanyl group, ticagrelor $C_{\max }$ did not significantly differ between groups (1,207 vs. $1,644 \mathrm{ng} / \mathrm{mL}$, $p=0.11$ ). Estimates for ticagrelor $T_{\max }$ were 3 hours $13 \mathrm{~min}$ utes for the fentanyl group and 2 hours 19 minutes for the nofentanyl group $(p=0.01)$.

\section{Extended Pharmacodynamic Results}

Platelet function differences were also larger in the astreated analyses, compared with the intention-to-treat 
Table 1 Patient and procedural characteristics $(N=70)^{\mathrm{a}}$

\begin{tabular}{|c|c|c|c|}
\hline & $\begin{array}{l}\text { No-fentanyl } \\
(N=35)\end{array}$ & $\begin{array}{l}\text { Fentanyl } \\
(N=35)\end{array}$ & $p$-Value \\
\hline \multicolumn{4}{|l|}{ Patient characteristics } \\
\hline Age, y & $65( \pm 9.2)$ & $61( \pm 11.7)$ & 0.13 \\
\hline Female & $13(38)$ & $7(20)$ & 0.10 \\
\hline \multicolumn{4}{|l|}{ Race } \\
\hline White & $27(77)$ & $25(71)$ & 0.92 \\
\hline Black & $3(9)$ & $3(9)$ & \\
\hline Other & $5(14)$ & $7(20)$ & \\
\hline $\begin{array}{l}\text { Body mass index, } \\
\mathrm{kg} / \mathrm{m}^{2}\end{array}$ & $29.6( \pm 5.4)$ & $29.9( \pm 4.2)$ & 0.79 \\
\hline Hypertension & $33(94)$ & $29(83)$ & 0.26 \\
\hline Diabetes mellitus & $14(40)$ & $7(20)$ & 0.12 \\
\hline Dyslipidaemia & $30(86)$ & $28(80)$ & 0.75 \\
\hline Current smoker & $5(16)$ & $2(6)$ & 0.24 \\
\hline History of MI & $5(14)$ & $6(17)$ & 1.00 \\
\hline $\begin{array}{l}\text { History of prior } \\
\text { PCI/CABG }\end{array}$ & $15(43)$ & $12(34)$ & 0.62 \\
\hline Home use of aspirin & $31(89)$ & $24(69)$ & 0.08 \\
\hline $\begin{array}{l}\text { Baseline platelet count, } \\
\mathrm{K} / \mathrm{cu} \mathrm{mm}\end{array}$ & $230( \pm 69)$ & $210( \pm 72)$ & 0.23 \\
\hline $\begin{array}{l}\text { Baseline haemoglobin, } \\
\mathrm{g} / \mathrm{dL}\end{array}$ & $13.9( \pm 1.1)$ & $14.5( \pm 1.5)$ & 0.07 \\
\hline Baseline INR & $1.05( \pm 0.12)$ & $1.13( \pm 0.34)$ & 0.20 \\
\hline $\begin{array}{l}\text { Baseline creatinine, } \\
\mathrm{mg} / \mathrm{dL}\end{array}$ & $0.95( \pm 0.22)$ & $0.99( \pm 0.27)$ & 0.36 \\
\hline \multicolumn{4}{|l|}{$\begin{array}{l}\begin{array}{l}\text { Procedural } \\
\text { characteristics }\end{array} \\
\end{array}$} \\
\hline $\begin{array}{l}\text { Initial fentanyl } \\
\text { dose, mcg }\end{array}$ & $0( \pm 0)$ & $27.5( \pm 8.5)$ & $<0.0001$ \\
\hline $\begin{array}{l}\text { Total fentanyl } \\
\text { dose, mcg }\end{array}$ & $9.3( \pm 3.5)$ & $96.3( \pm 5.2)$ & $<0.0001$ \\
\hline $\begin{array}{l}\text { Total midazolam } \\
\text { dose, } \mathrm{mg}\end{array}$ & $2.3( \pm 2.0)$ & $2.5( \pm 1.6)$ & 0.71 \\
\hline $\begin{array}{l}\text { Total intra-arterial } \\
\text { nicardipine, mcg }\end{array}$ & $422( \pm 352)$ & $363( \pm 529)$ & 0.64 \\
\hline $\begin{array}{l}\text { Total intra-arterial } \\
\text { NTG, mEq }\end{array}$ & $252( \pm 239)$ & $184( \pm 234)$ & 0.29 \\
\hline Radial access & $27(77)$ & $23(66)$ & 0.43 \\
\hline $\begin{array}{l}\text { Intra-procedural } \\
\text { GPIIb/IIla-antagonist }\end{array}$ & $0(0)$ & $1(3)$ & 1.00 \\
\hline $\begin{array}{l}\text { Intra-procedural } \\
\text { intravenous } \\
\text { unfractionated heparin }\end{array}$ & $35(100)$ & $35(100)$ & 1.00 \\
\hline
\end{tabular}

Abbreviations: CABG, coronary artery bypass grafting; GPIIb/IIla, glycoprotein 2b3a inhibitor; INR, international normalized ratio; $\mathrm{MI}$, myocardial infarction; NTG, nitroglycerin; PCl, percutaneous coronary intervention; SD, standard deviation.

Note: Values are $n(\%)$ or mean \pm SD.

antention-to-treat analysis.

data previously reported. For example, the 2-hour mean (standard deviation [SD]) PRU value was $113( \pm 94)$ in the fentanyl group compared with $71( \pm 66)$ in the no-fentanyl group ( $p=0.03$ ). This finding was highly significant for ADP response measured using platelet aggregometry (fentanyl
$40.6 \%$ [ \pm 19.2$]$ vs. no-fentanyl 25.2\% [ \pm 10.8$], p=0.004)$. These differences yielded significantly higher rates of 2-hour HPR in the fentanyl group compared with those who did not receive fentanyl ( $20 \%$ vs. $3 \%$ by PRU [ $p=0.03$ ] and $36 \%$ vs. $0 \%$ by aggregometry [ $p=0.004]$ ) ( - Fig. 3). Results using a PRU cut-off of $\geq 208$ for HPR were similar (-Supplementary Table S2, available in the online version).

The PRU values and proportion with HPR at each of the study time points are presented in -Table $\mathbf{2}$ and -Supplementary Fig. $\mathbf{5 2}$ (available in the online version). PRU values were in the normal range in both groups at baseline and 0.5 hours. At 1 hour, the mean PRU value was $11 \%$ higher in the fentanyl group, but not significantly so. At 2 hours, the mean PRU was roughly 60\% higher in the fentanyl $\operatorname{arm}(p=0.02)$. By 4 and 24 hours, PRU values were similarly low in both arms.

\section{High-Sensitivity Troponin}

Mean (SD) hs-TnI level 2 hours post-PCI was higher in persons randomized to fentanyl, in both intention-to-treat (12.1 [9.5] vs. 6.8 [4.2] ng/L, $p=0.02$ ) and as-treated (11.9 [10.4] vs. 7.0 [4.2] ng/L, $p=0.04$ ) analyses (-Supplementary Fig. S3, available in the online version). The 99th percentile for this hs-TnI assay is $26.2 \mathrm{ng} / \mathrm{L}$ and, in both intention-totreat and as-treated analyses, $11 \%$ of those randomized to fentanyl for PCI exceeded this threshold whereas $0 \%$ of those randomized to no-fentanyl did ( $p=0.08$ ). Results for hs-cTnI values at 4 hours post-PCI were available for a non-random sub-group of 38 participants who had PCI. The mean 4 hour hs-TnI level was $16 \mathrm{ng} / \mathrm{L}$ in the fentanyl group versus $10 \mathrm{ng} / \mathrm{L}$ in the no-fentanyl group $(p=0.12)$.

\section{Full Report of Patient Comfort Outcomes}

All 212 participants enrolled in the study, including the 70 who underwent $\mathrm{PCI}$ and the 142 who were consented but did not require PCI after coronary angiography, completed the nurse-administered pain and anxiety survey 2 hours after the end of the procedure (-Table 3 ). Mean self-reported maximal intra-procedural pain was $1.5 / 10$ in the fentanyl arm versus 2.3/10 in the no-fentanyl arm, but the difference was not statistically significant $(p=0.14)$. The number of participants who reported significant pain $(>5 / 10)$ was $7 /$ 107 in the fentanyl arm versus 13/105 in the no-fentanyl arm $(p=0.13)$. There were also no significant differences in mean procedural pain when the analysis was limited to those who underwent $\mathrm{PCI}$ (2.3 vs. $2.8 / 10, p=0.46$ ), or among those who had femoral access for PCI. No differences in pain were reported at the 2-hour post-procedure mark either. Nurse-documented maximal pain recorded in the electronic health record was also not different between the fentanyl and no-fentanyl arms. All of the patient comfort results were nearly identical in the intention-to-treat and per protocol (as-treated) analyses.

\section{Discussion}

The PACIFY study demonstrates that fentanyl significantly impairs the rapid absorption and action of oral ticagrelor, 


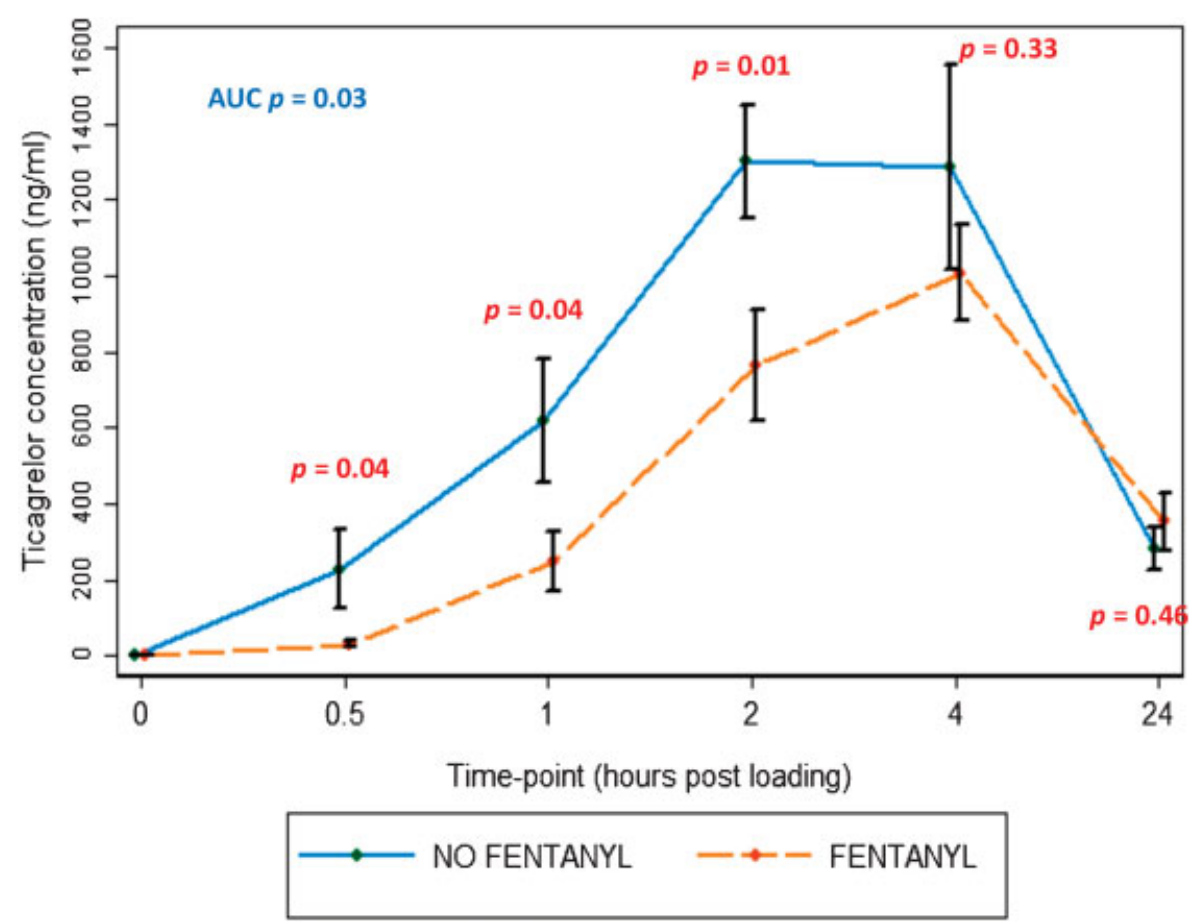

Fig. 2 Pharmacokinetic results. *Plasma concentrations (means and standard errors) of ticagrelor after a 180-mg oral load, according to fentanyl administration $(\mathrm{N}=70)$. $p$-Values in red are for differences in mean ticagrelor concentration at each time point. *As-treated analysis.

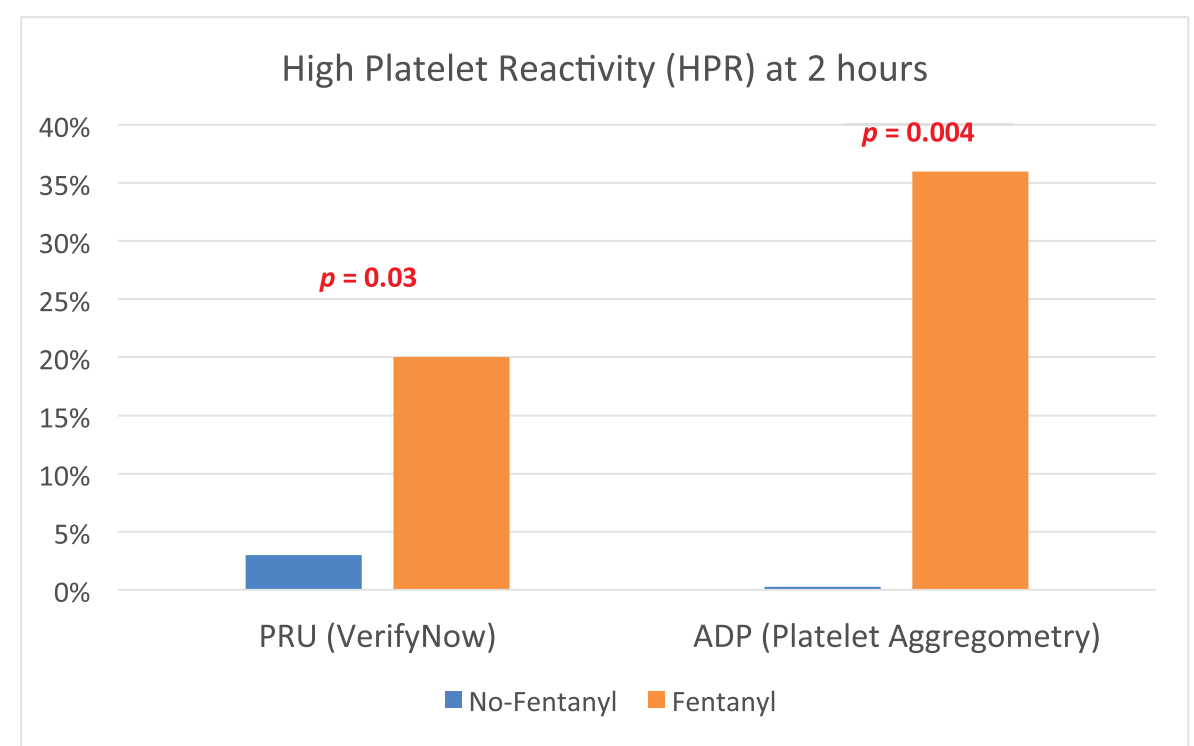

Fig. 3 High platelet reactivity 2 hours after loading. *High platelet reactivity 2 hours after $180 \mathrm{mg}$ ticagrelor loading based on fentanyl administration, assessed by both VerifyNow (PRU $\geq 235$ ) and light-transmission aggregometry ( $\geq 46 \%$ change from baseline). *As-treated analysis ( $N=69$ after excluding one participant who received a GPIIb/IIla antagonist). PRU $=$ P2 $\mathrm{Y}_{12}$ reaction units (measured by VerifyNow), $\mathrm{ADP}=$ response to adenosine diphosphate.

an important platelet inhibition therapy that requires gastric emptying for absorption in the intestines. ${ }^{14,20}$ Ticagrelor concentrations peaked at 2 hours in the nofentanyl group versus approximately 4 hours in the fentanyl group. Platelet inhibition was more complete in the no-fentanyl arm at 2 hours when assessed using two independent testing modalities. Both groups had similar mean platelet inhibition by 4 hours, as measured by PRUs, reflecting eventual though delayed absorption of the loading dose. 
Table 2 Mean P2Y 12 reaction units (PRU, VerifyNow) at multiple time points after ticagrelor loading, with HPR proportions at 1, 2, 4 and 24 hours $(N=69)^{a}$

\begin{tabular}{|l|l|l|l|}
\hline & $\begin{array}{l}\text { No-fentanyl } \\
(\boldsymbol{n}=33)\end{array}$ & $\begin{array}{l}\text { Fentanyl } \\
(\boldsymbol{n}=\mathbf{3 6})\end{array}$ & $p$-Value \\
\hline PRU 0 h & 283.7 & 270.2 & 0.26 \\
\hline PRU 0.5 h & 252.9 & 250.6 & 0.89 \\
\hline PRU 1 h & 182.4 & 201.0 & 0.44 \\
\hline \% HPR 1 h & $36 \%$ & $54 \%$ & 0.10 \\
\hline PRU 2 h & 70.6 & 112.9 & 0.03 \\
\hline \% HPR 2 h & $3 \%$ & $20 \%$ & 0.03 \\
\hline PRU 4 h & 50.7 & 54.0 & 0.82 \\
\hline \% HPR 4 h & $0 \%$ & $6 \%$ & 0.17 \\
\hline PRU 24 h & 84.0 & 64.2 & 0.51 \\
\hline \% HPR 24 h & $1 \%$ & $1 \%$ & 0.91 \\
\hline
\end{tabular}

Abbreviation: HPR $=$ high platelet reactivity on treatment, PRU $\geq 235$. aPer-protocol (as-treated) analysis, which includes two cross-overs from the no-fentanyl to the fentanyl arm (note also that 1 participant from the fentanyl arm who received a GPIIb/IIla-antagonist was excluded from these platelet function analyses).
This full reporting of the PACIFY trial results also demonstrates that the effects of fentanyl on ticagrelor concentration and platelet function were even more pronounced when participants were analysed according to the treatment received. These as-treated analyses complement the previously reported intention-to-treat analyses, ${ }^{14}$ both because the effect of fentanyl on the primary and secondary outcomes was even more significant than originally reported and because the likelihood of crossover from no-fentanyl to fentanyl biasing our pharmacokinetic and pharmacodynamics outcomes is low. In addition, this report extends knowledge on the impact of fentanyl on post-PCI procedural troponin levels (numerically higher values at 2 and 4 hours post-PCI with more post-procedural enzymatic myocardial infarctions), although this finding is hypothesis generating given the small sample size and warrants further study. Finally, the extended results for self-reported patient comfort outcomes are important because they provide further reassurance that restricting the use of fentanyl to the breakthrough treatment of pain does not expose patients to excess discomfort during coronary angiography. This would represent a clinical change from the widely employed strategy (at least in the United States) of

Table 3 Patient comfort ${ }^{\mathrm{a}}$ : Overall $(N=212)$ among those who underwent PCI $(n=70 / 212)$ and among femoral access cases $(n=35 / 212)^{\mathrm{b}}$

\begin{tabular}{|c|c|c|c|c|}
\hline & $\begin{array}{l}\text { No-fentanyl } \\
(n=105)\end{array}$ & $\begin{array}{l}\text { Fentanyl } \\
(n=107)\end{array}$ & $\begin{array}{l}\text { Mean } \\
\text { difference } \\
(95 \% \mathrm{Cl})\end{array}$ & $p$-Value \\
\hline Self-reported procedural anxiety, mean ( \pm SD) & $2.2( \pm 2.8)$ & $2.0( \pm 2.1)$ & $0.2(-0.6,0.9)$ & 0.63 \\
\hline Self-reported maximal procedural pain, mean $( \pm S D)$ & $2.3( \pm 3.1)$ & $1.5( \pm 2.3)$ & $0.8(-0.1,1.6)$ & 0.14 \\
\hline Self-reported maximal procedural pain $>5, n(\%)$ & $13(12 \%)$ & $7(7 \%)$ & $5 \%(-3 \%, 15 \%)^{c}$ & 0.13 \\
\hline Self-reported pain at $2 \mathrm{~h}$, mean ( $\pm \mathrm{SD})$ & $0.6( \pm 1.3)$ & $0.5( \pm 1.3)$ & $0.1(-0.3,0.5)$ & 0.44 \\
\hline Nurse-documented maximum procedural pain, mean ( \pm SD) & $0.8( \pm 2.1)$ & $0.5( \pm 1.6)$ & $0.3(-0.2,0.8)$ & 0.22 \\
\hline \multicolumn{5}{|l|}{$\mathrm{PCl}$ cases $(n=70)$} \\
\hline Self-reported procedural anxiety, mean ( \pm SD) & $2.6( \pm 2.7)$ & $2.5( \pm 2.1)$ & $0.1(-1.2,1.4)$ & 0.87 \\
\hline Self-reported maximal procedural pain, mean $( \pm S D)$ & $2.8( \pm 2.9)$ & $2.3( \pm 2.9)$ & $0.5(-0.9,2.0)$ & 0.46 \\
\hline Self-reported maximal procedural pain $>5, n(\%)$ & $6(19 \%)$ & $6(18 \%)$ & $1 \%(-18 \%, 20 \%)^{c}$ & 0.90 \\
\hline Self-reported pain at $2 \mathrm{~h}$, mean $( \pm$ SD) & $0.8( \pm 1.8)$ & $0.7( \pm 1.6)$ & $0.1(-0.7,0.9)$ & 0.77 \\
\hline Nurse-documented maximum procedural pain, mean ( \pm SD) & $1.3( \pm 2.6)$ & $0.9( \pm 2.3)$ & $0.4(-0.8,1.6)$ & 0.51 \\
\hline \multicolumn{5}{|l|}{ Femoral cases $(n=35)$} \\
\hline Self-reported procedural anxiety, mean ( \pm SD) & $2.2( \pm 3.1)$ & $2.6( \pm 2.1)$ & $-0.4(-2.2,1.4)$ & 0.65 \\
\hline Self-reported maximal procedural pain, mean $( \pm S D)$ & $2.8( \pm 3.2)$ & $2.3( \pm 3.1)$ & $0.5(-1.6,2.6)$ & 0.65 \\
\hline Self-reported maximal procedural pain $>5, n(\%)$ & $4(22 \%)$ & $4(22 \%)$ & $0 \%(-27 \%, 27 \%)^{c}$ & 1.00 \\
\hline Self-reported pain at $2 \mathrm{~h}$, mean $( \pm S D)$ & $0.4( \pm 0.8)$ & $0.5( \pm 1.0)$ & $-0.1(-0.7,0.6)$ & 0.86 \\
\hline Nurse-documented maximum procedural pain, mean ( \pm SD) & $0.5( \pm 1.5)$ & $0.6( \pm 1.8)$ & $-0.1(-1.2,1.1)$ & 0.88 \\
\hline
\end{tabular}

Abbreviations: $\mathrm{Cl}$, confidence interval; $\mathrm{PCl}$, percutaneous coronary intervention; SD, standard deviation.

${ }^{a}$ Numerical scale of $0-10$, lower values signify less pain or anxiety.

${ }^{\mathrm{b}}$ As-treated analysis.

'Difference in proportions (\%) with pain score > 5: all other values are for differences in mean score on the 0-10 scale. 
administering fentanyl prophylactically at the start of angiography and as needed during the case (with pro re nata fentanyl administration also not infrequently occurring for non-pain symptoms like anxiety).

The PACIFY trial findings are consistent with prior knowledge about the effect of other intravenous opioids on oral $\mathrm{P}_{2} \mathrm{Y}_{12}$ absorption. Hobl et al demonstrated in healthy volunteers that intravenous morphine delays the absorption of clopidogrel and impairs its inhibition of platelets. ${ }^{4}$ The findings of Hobl et al, and others, ${ }^{7}$ help to explain, at least in part, the long-established association between morphine and adverse outcomes in ACS. ${ }^{2,12}$ However, fentanyl is far more short acting than morphine and, while we assumed for sample size purposes the effect of fentanyl on $\mathrm{P}_{2} \mathrm{Y}_{12}$ inhibitors may be similar to morphine, the PACIFY trial results confirm that this assumption was correct. Importantly, the PACIFY trial also highlights the common misconception that fentanyl can be used instead of morphine to avoid this opiate-related drug interaction.

For the secondary endpoints of safety and patient comfort, our results are consistent with prior studies. Baris et al randomized 90 patients undergoing coronary angiography to either midazolam and fentanyl, midazolam and placebo or double placebo, and found no differences in sedation scores, anxiolysis and patient and cardiologist satisfaction between the first two comparisons. ${ }^{23}$ Kennedy et al randomized 125 patients undergoing aortofemoral angiography to either placebo, oral temazepam, intravenous midazolam or intravenous midazolam and fentanyl, and found similar self-reported pain scores for the midazolam and midazolam + fentanyl groups (1.5 vs. 1.3 on a 5-point pain scale, $p$-value not reported). ${ }^{24}$ We found a marginally lower numerical pain level in those who received fentanyl in the full PACIFY sample (2.3 vs. 1.5 on a 10-point scale, $p=0.14$ ); although the absolute difference was small, statistically non-significant and of uncertain clinical significance. Furthermore, it is reassuring that pain levels were almost identical between the groups among PACIFY participants who had PCI and those who had femoral vascular access for the catheterization, both of which are typically more uncomfortable than simple radial coronary angiography.

These trial data have several implications. To start, the PACIFY trial describes an important drug-drug interaction that could have clinical implications for certain patients in the catheterization laboratory. For both elective and especially emergent PCI cases, our study suggests that nonselective use of fentanyl perhaps should be avoided in the absence of pain, particularly when rapid platelet inhibition is desired. U.S. interventional cardiologists may want to closely scrutinize whether fentanyl is required, monitor the dose provided and limit non-selective administration. These data will also be of interest to emergency room providers who are considering fentanyl administration in chest-pain patients proceeding directly to $\mathrm{PCI}$. However, we cannot advocate for withholding this medication where clinically necessary for the treatment of pain. If pre- or intra-procedural opioids are required, given delayed oral platelet inhibition, there may be a role in certain clinical circumstances (e.g. cases deemed high risk for stent thrombosis) for short-term bridging with a parenteral $\mathrm{P}_{2} \mathrm{Y}_{12}$ inhibitors such as cangrelor, ${ }^{25,26}$ or other parenteral alternatives; a strategy that warrants future study.

This study inevitably has limitations. It was not powered for clinical outcomes and larger studies would be needed to evaluate the effect of fentanyl-ticagrelor co-administration on these endpoints (e.g. stent thrombosis). Based on the approximately 2-hour delay in effective platelet inhibition, it is probable that the magnitude of any clinical effect may be small in frequency, but for the patients who suffer stent thrombosis the clinical consequences can be catastrophic. We did not power this study for pain outcomes either, so, while the numerical differences in subject comfort scores were small between the groups, further studies would be required to determine whether these small differences are significant statistically. We could not blind catheterization laboratory nurses and doctors to the randomized treatment, so some patients may have become aware of treatment assignment during the case. All patients received midazolam, which can cause post-procedural amnesia and could affect patient-reported intra-procedural pain and anxiety. However, objective nurse reports of intra-procedural pain were also no different between the groups. We used uncrushed ticagrelor, which is a common practice. The effect of fentanyl on crushed ticagrelor may warrant further study. ${ }^{15}$ We excluded $<10 \%$ of the pharmacokinetic data from the AUC analysis, specifically, 24-hour ticagrelor concentrations from those participants who received a ticagrelor maintenance dose between the 4-hour and 24-hour laboratory draw; however, such exclusions were balanced between the randomized groups and would not have affected the early differences in blood concentration (between 30 minutes and 4 hours) found in this study. Finally, while the PACIFY results may be most important in the setting of unstable ACS patients (where $\mathrm{P}_{2} \mathrm{Y}_{12}$ pretreatment is not possible and where ticagrelor is most commonly administered), our study was limited to more stable PCI patients. However, we see no a priori reason why fentanyl's effects on P2Y $\mathrm{Y}_{12}$ absorption would be weaker in unstable patients, indeed prior data indicate they may be stronger. ${ }^{5,7}$

In conclusion, routine use of fentanyl for PCI sedation delays ticagrelor absorption and platelet inhibition in CAD patients, resulting in mild excess of post-PCI myocardial damage; all without significant evidence of substantial improvement in subjective comfort (which was well controlled in both arms of this study in the presence of local anaesthetic and sedation by midazolam). This represents a newly recognized drug-drug interaction with potential clinical implications that warrant further study. Particularly on a background of less common use of fentanyl in non-U.S. catheterization laboratories, ${ }^{22}$ these data suggest also that the often routine and non-selective use of fentanyl during cardiac catheterization and PCI may need to be reconsidered; particularly in the absence of pain and when rapid platelet inhibition is desirable. 


\section{What is known about this topic?}

- Morphine use has been associated with adverse outcomes in patients with acute coronary syndrome.

- Randomized trials have demonstrated that, by slowing gastrointestinal absorption, morphine can delay and reduce the effects of oral $\mathrm{P}_{2} \mathrm{Y}_{12}$ platelet inhibitors like clopidogrel, ticagrelor and prasugrel.

- Fentanyl is a more potent and short acting opioid that is routinely used in many U.S. catheterization laboratories for pain prophylaxis and treatment among cardiac patients undergoing coronary angiography and stenting. However, fentanyl is infrequently used in many non-U.S. (e.g. European) catheterization laboratories and it is unknown if, like morphine, fentanyl also

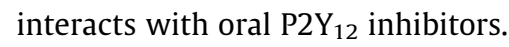

\section{What does this paper add?}

- This article provides a detailed reporting of the full complement of primary and secondary outcome results from the PACIFY (Platelet Aggregation with tiCagrelor Inhibition and FentanYl) randomized trial (NCT02683707).

- Fentanyl delays the absorption of ticagrelor and results in less inhibited platelets at 30 minutes, 1 hour and 2 hours after coronary stenting. By 4 hours, both arms of the study had inhibited platelets. However, the 2and 4-hour troponin levels were higher with fentanyl, suggesting a clinically important drug interaction and motivating larger studies evaluating stent thrombosis outcomes.

- Pain was well controlled in both the fentanyl and nofentanyl arms of this study, with no statistical differences between groups. While we cannot argue against judicious fentanyl use where necessary for pain control, our results support the European standard of not routinely providing potent opiates for cardiac procedures where rapid platelet inhibition is important.

\section{Authors' Contributions}

J.W.M. conceived the study, designed the trial and obtained research funding. J.W.M., S.P.S. and J.R.R. supervised the conduct of the trial and data collection. K.I., T.S. K. and W.A.C. undertook recruitment of participating centres and patients and managed the data, including quality control. S.P.S., J.R.R., D.R.T. and R.K.H. provided statistical advice on study design and analysed the data. K. I. and R.S. drafted the manuscript, and all authors contributed substantially to its revision. J.W.M. takes responsibility for the article as a whole.

Funding

This study was supported by the Johns Hopkins Magic That Matters Research Grant.

\section{Conflict of Interest}

None.

\section{Acknowledgments}

The investigators thank the patients who participated in this study for their critical contribution. Drs. McEvoy and Schulman thank their research coordinator, Ms. Frances A. Kirkland, without whom this study would not have been possible. PACIFY was fully funded by an institutional grant to Dr. McEvoy from The Johns Hopkins Magic That Matters Research Grant.

\section{References}

1 Lavi S, Jolly SS, Bainbridge D, Manji F, Randhawa V, Lavi R. Sedation, analgesia, and anaesthesia variability in laboratorybased cardiac procedures: an international survey. Can J Cardiol 2014;30(06):627-633

2 McCarthy CP, Mullins KV, Sidhu SS, Schulman SP, McEvoy JW. The on- and off-target effects of morphine in acute coronary syndrome: a narrative review. Am Heart J 2016;176:114-121

3 Kolh P, Windecker S, Alfonso F, et al; European Society of Cardiology Committee for Practice Guidelines; EACTS Clinical Guidelines Committee; Task Force on Myocardial Revascularization of the European Society of Cardiology and the European Association for Cardio-Thoracic Surgery; European Association of Percutaneous Cardiovascular Interventions. 2014 ESC/EACTS Guidelines on myocardial revascularization: the Task Force on Myocardial Revascularization of the European Society of Cardiology (ESC) and the European Association for Cardio-Thoracic Surgery (EACTS). Developed with the special contribution of the European Association of Percutaneous Cardiovascular Interventions (EAPCI). Eur J Cardiothorac Surg 2014;46(04):517-592

4 Hobl E-L, Stimpfl T, Ebner J, et al. Morphine decreases clopidogrel concentrations and effects: a randomized, double-blind, placebocontrolled trial. J Am Coll Cardiol 2014;63(07):630-635

5 Hobl EL, Reiter B, Schoergenhofer C, et al. Morphine decreases ticagrelor concentrations but not its antiplatelet effects: a randomized trial in healthy volunteers. Eur J Clin Invest 2016; 46(01):7-14

6 Hobl E-L, Reiter B, Schoergenhofer C, et al. Morphine interaction with prasugrel: a double-blind, cross-over trial in healthy volunteers. Clin Res Cardiol 2016;105(04):349-355

7 Kubica J, Adamski P, Ostrowska M, et al. Morphine delays and attenuates ticagrelor exposure and action in patients with myocardial infarction: the randomized, double-blind, placebocontrolled IMPRESSION trial. Eur Heart J 2016;37(03):245-252

8 Thomas MR, Morton AC, Hossain R, et al. Morphine delays the onset of action of prasugrel in patients with prior history of STelevation myocardial infarction. Thromb Haemost 2016;116(01): 96-102

9 Silvain J, Storey RF, Cayla G, et al. P2Y12 receptor inhibition and effect of morphine in patients undergoing primary PCI for STsegment elevation myocardial infarction. The PRIVATE-ATLANTIC study. Thromb Haemost 2016;116(02):369-378

10 Farag M, Spinthakis N, Srinivasan M, Sullivan K, Wellsted D, Gorog DA. Morphine analgesia pre-PPCI is associated with prothrombotic state, reduced spontaneous reperfusion and greater infarct size. Thromb Haemost 2018;118(03):601-612

11 Nimmo WS, Heading RC, Wilson J, Tothill P, Prescott LF. Inhibition of gastric emptying and drug absorption by narcotic analgesics. $\mathrm{Br}$ J Clin Pharmacol 1975;2(06):509-513

12 Meine TJ, Roe MT, Chen AY, et al; CRUSADE Investigators. Association of intravenous morphine use and outcomes in acute coronary syndromes: results from the CRUSADE Quality Improvement Initiative. Am Heart J 2005;149(06):1043-1049 
13 Ibrahim K, Goli RR, Shah R, Resar JR, Schulman SP, McEvoy JW. Effect of intravenous fentanyl on ticagrelor absorption and platelet inhibition among patients undergoing percutaneous coronary intervention: design, rationale, and sample characteristics of the PACIFY randomized trial. Contemp Clin Trials 2018;64:8-12

14 McEvoy JW, Ibrahim K, Kickler TS, et al. Effect of intravenous fentanyl on ticagrelor absorption and platelet inhibition among patients undergoing percutaneous coronary intervention: the PACIFY Randomized Clinical Trial (Platelet Aggregation With Ticagrelor Inhibition and Fentanyl). Circulation 2018;137(03):307-309

15 Parodi G, Xanthopoulou I, Bellandi B, et al. Ticagrelor crushed tablets administration in STEMI patients: the MOJITO study. J Am Coll Cardiol 2015;65(05):511-512

16 Angiolillo DJ, Franchi F, Waksman R, et al. Effects of ticagrelor versus clopidogrel in troponin-negative patients with low-risk ACS undergoing ad hoc PCI. J Am Coll Cardiol 2016;67(06):603-613

17 Rollini F, Franchi F, Angiolillo DJ. Switching P2Y12-receptor inhibitors in patients with coronary artery disease. Nat Rev Cardiol 2016;13(01):11-27

18 Breet NJ, van Werkum JW, Bouman HJ, et al. Comparison of platelet function tests in predicting clinical outcome in patients undergoing coronary stent implantation. JAMA 2010;303(08):754-762

19 Price MJ, Endemann S, Gollapudi RR, et al. Prognostic significance of post-clopidogrel platelet reactivity assessed by a point-of-care assay on thrombotic events after drug-eluting stent implantation. Eur Heart J 2008;29(08):992-1000

20 Teng R, Maya J. Absolute bioavailability and regional absorption of ticagrelor in healthy volunteers. J Drug Assess 2014;3(01):43-50

$21 \mathrm{Li}$ P, Gu Y, Yang Y, et al. Low-dose ticagrelor yields an antiplatelet efficacy similar to that of standard-dose ticagrelor in healthy subjects: an open-label randomized controlled trial. Sci Rep 2016; 6:31838

22 Shah R, Kirtane AJ, McEvoy JW. Opiate use in the cath lab. Eur Heart J 2018;39(08):642-645

23 Baris S, Karakaya D, Aykent R, Kirdar K, Sagkan O, Tür A. Comparison of midazolam with or without fentanyl for conscious sedation and hemodynamics in coronary angiography. Can J Cardiol 2001;17(03):277-281

24 Kennedy PT, Kelly IM, Loan WC, Boyd CS. Conscious sedation and analgesia for routine aortofemoral arteriography: a prospective evaluation. Radiology 2000;216(03):660-664

25 Bhatt DL, Lincoff AM, Gibson CM, et al; CHAMPION PLATFORM Investigators. Intravenous platelet blockade with cangrelor during PCI. N Engl J Med 2009;361(24):2330-2341

26 Angiolillo DJ, Firstenberg MS, Price MJ, et al; BRIDGE Investigators. Bridging antiplatelet therapy with cangrelor in patients undergoing cardiac surgery: a randomized controlled trial. JAMA 2012; 307(03):265-274 